\title{
FLEXIBILITY AND THE DEMAND FOR RISKY ASSETS
}

\author{
Mark J. MACHINA* \\ Princeton University, Princeton, NJ 08544, USA \\ University of California, San Diego, CA 92110, USA
}

Received 22 March 1982

The fact that reduced flexibility always increases the demand for insurance has led to the belief that reduced flexibility always makes individuals more 'risk averse'. However, it is quite possible for reduced flexibility to lead to less risk averse behavior in the standard asset demand sense.

To a decisionmaker facing a choice over a set of risky prospects the issue of 'flexibility", i.e., the ability to postpone other decisions until after the uncertainty is resolved, is a crucial one. Tisdell (1963), for example, has shown that the well known 'preference' of a risk neutral firm for random over constant prices (with the same mean) reduces to indifference when it is assumed that the firm must make its production decisions before the random prices are realized. In the literature, risky prospects which are due to be resolved before any other decisions must be made are known as 'timeless' prospects and prospects which are due to be resolved only after (some) such decisions must be made are known as 'temporal' prospects. '

* I am indebted to Mike Katz and Richard Zeckhauser for helpful discussions on this material.

I See for example Markowitz (1959, chs. 10, 11). Mossin (1969). Spence and Zeckhauser (1972), Kreps and Porteus (1979), Epstein (1980), and Rossman and Selden (1979). Note that 'timeless' and 'temporal' are not properties of prospects per se but rather of the decision maker's situation, so that the same prospect may be timeless for one individual and temporal for another.

0165-1765/82/0000-0000/\$02.75 (C) 1982 North-Holland 
To see the importance of this distinction, consider an individual who must choose over a set of alternative risky prospects $\left\{\tilde{x}_{i}\right\}$ with respective cumulative distribution functions $\left\{F_{i}(\cdot)\right\}$ as well as make some other choice $\alpha$ out of a set $A$, and who seeks to maximize the expectation of a von Neumann-Morgenstern utility function $U(x, \alpha)$. In the 'flexible' or 'timeless risk' case when $\alpha$ can be chosen after $\tilde{x}$ is realized, choosing a distribution $F(\cdot)$ allows the individual to attain an expected utility of $W(F) \equiv \int \max _{\alpha \in A}[U(x, \alpha)] \mathrm{d} F(x)$. However, in the 'inflexible' or 'temporal risk' case where $\alpha$ must be chosen before $\tilde{x}$ is realized, choosing a distribution $F(\cdot)$ only allows the individual to attain an expected utility of $V(F) \equiv \max _{\alpha \in A}\left[\int U(x, \alpha) \mathrm{d} F(x)\right]$, which can never be greater than $W(F)$. Since the functionals $W(\cdot)$ and $V(\cdot)$ will typically not be ordinally equivalent, it follows that the individual's preference ranking over temporal prospects will in general be quite distinct from his or her preference ranking over timeless prospects.

One of the fundamental results in the literature on flexibility and temporal risk is that, provided $U$ is increasing in $x$, greater flexibility will never raise the premium that the individual would be willing to pay for insurance against a given risky prospect, so that if $\pi_{F}$ ('flexible') and $\pi_{I}$ ('inflexible') solve $\max _{\alpha \in A}\left[U\left(\bar{x}-\pi_{F}, \alpha\right)\right]=\int \max _{\alpha \in A}[U(x, \alpha)] \mathrm{d} F(x)$ and $\max _{\alpha \in A}\left[U\left(\bar{x}-\pi_{I}, \alpha\right)\right]=\max _{\alpha \in A}\left[\int U(x, \alpha) \mathrm{d} F(x)\right]$ for some distribution $F(\cdot)$ with mean $\bar{x}$, then $\pi_{F} \leqslant \pi_{I}{ }^{2}$ In other words, the insurance premia derived from the individual's ranking over temporal prospects [i.e., from $V(\cdot)$ ] will always be at least as great as those derived from the individual's ranking over timeless prospects [i.e., from $W(\cdot)$ ]. In particular, note that this result does not depend upon the assumption of risk aversion (i.e., $U$ concave in $x$ ) but rather applies to any utility function $U(x, \alpha)$ provided it is increasing in $x$ and that the relevant maxima exist. ${ }^{3}$

Not surprisingly, this result has often been taken to imply that reduced flexibility leads to a greater aversion to risk, or in other words

2 See for example Drèze and Modigliani (1972) and Spence and Zeckhauser (1972). To derive this result, assume $\pi_{F}>\pi_{I}$ and let $\alpha_{F}$ be a solution to $\max _{\alpha \in A}\left[U\left(\bar{x}-\pi_{F}, \alpha\right)\right]$. Then strict monotonicity of $U$ in $x$ and the definition of $\pi_{F}$ and $\pi_{I}$ imply $W(F)=$ $\int \max _{\alpha \in A}[U(x, \alpha)] \mathrm{d} F(x)=\max _{\alpha \in A}\left[U\left(\bar{x}-\pi_{F}, \alpha\right)\right]=U\left(\bar{x}-\pi_{F}, \alpha_{F}\right)<U\left(\bar{x}-\pi_{I}, \alpha_{F}\right) \leqslant$ $\max _{\alpha \in A_{A}}\left[U\left(\bar{x}-\pi_{I}, \alpha\right)\right]=\max _{\alpha \in A}\left[\int U(x, \alpha) \mathrm{d} F(x)\right]=V(F)$, which contradicts the fact that $V(F)$ can be no greater than $W(F)$.

${ }^{3}$ See Drèze and Modigliani (1972) and Eden (1977). In the case when $U$ is convex in $x$ the inequality $\pi_{F} \leqslant \pi_{I} \leqslant 0$ implies that the individual would pay more to obtain a timeless prospect than an identically distributed temporal one. 
that an individual's preferences over temporal prospects display a higher level of 'risk aversion' than his or her preferences over timeless prospects. Spence and Zeckhauser (1972, p. 402) for example, claim that to an external observer unaware of the distinction, an individual 'will appear to be substantially more risk averse' when choosing over temporal prospects than when choosing over a set of identically distributed timeless prospects. In the special case of a two period consumption/savings model, Drèze and Modigliani (1972) similarly derive a local measure of aversion to temporal risk ('local' in the sense of applying only to infinitesimal risks) and show that it will always be at least as great as their corresponding local measure of timeless risk aversion.

In classic contributions to the theory of risk bearing, Arrow (1974) and Pratt (1964) have shown that the condition that one expected utility maximizer would always pay at least as much for insurance as another is equivalent to the condition that, in a world with one riskless and one risky asset, the first individual would never buy more of the risky asset than would the second. The equivalence of these two conditions is of course what makes them a natural definition of the relation 'more risk averse than', and in light of the above result on temporal vs. timeless insurance premia, we might therefore expect that an individual would always purchase at least as much of the risky asset when its risk is timeless than when it is temporal, or in other words, that greater flexibility would never lower the individual's demand for the risky asset.

It is the purpose of this note, however, to demonstrate that the characterization of preferences over temporal prospects as more 'risk averse' than preferences over timeless prospects does not extend to the case of asset demand behavior. Rather, and perhaps surprisingly, it is quite possible for a reduction in flexibility to induce the individual to take a riskier asset position by reallocating investment funds out of the riskless and into the risky asset.

To see this, consider the following example: ${ }^{4}$ an individual must allocate a given amount of wealth between a riskless asset with (gross) return $R$ per share and a risky asset with return $\tilde{z}$ per share, and will use the proceeds to purchase $\alpha$ units of housing and $c$ units of consumption, in order to maximize the expectation of $\xi(c, \alpha) \equiv-\exp [-k \alpha(c+\alpha-$ $M)$ ). Housing is available in two discrete size levels $\alpha_{0}$ and $\alpha_{1} ; \tilde{z}$ takes on the values $R+S$ and $R-1$ with equal probability; the prices of both

4 I am grateful to Richard Zeckhauser for providing me with an alternative example which makes the same point. 
housing and consumption are unity; and the individual has just enough wealth to purchase one share of the riskless asset, one share of the risky asset, or any convex combination of the two. In particular, let $R=20$, $S=8, k=0.06, M=21, \alpha_{0}=11$, and $\alpha_{1}=19 .{ }^{5}$ Substituting in the ex post budget constraint $c+\alpha=x$ yields the 'indirect' utility function $U(x, \alpha) \equiv \xi(x-\alpha, \alpha)=-\exp [-k \alpha(x-M)]$ in terms of housing services $\alpha$ and the ex post value of the portfolio $x$.

In the flexible or timeless risk case when $\alpha$ can be chosen after $\tilde{x}$ is known, the optimal proportion of wealth to invest in the risky asset will be that $\beta$ which yields the highest value of

$$
\begin{aligned}
& \int \max _{\left\{\alpha_{0}, \alpha_{1}\right\}}[U(R+\beta z, \alpha)] \mathrm{d} F(z) \\
& \quad=\frac{1}{2}\left[\max _{\left\{\alpha_{0}, \alpha_{1}\right\}}[U(R+\beta S, \alpha)]+\max _{\left\{\alpha_{0}, \alpha_{1}\right\}}[U(R-\beta, \alpha)]\right] .
\end{aligned}
$$

For the given values of the parameters, the individual will maximize by choosing $\beta_{F} \approx 0.318$ and buying $\alpha_{1}$ units of housing if the risky asset yields the high return and $\alpha_{0}$ units if it yields the low return.

In the inflexible or temporal risk case where $\alpha$ must be chosen ex ante, the individual will choose $\beta$ to maximize the value of

$$
\begin{aligned}
& \max _{\left\{\alpha_{0}, \alpha_{1}\right\}}\left[\int U(R+\beta z, \alpha) \mathrm{d} F(z)\right] \\
& \quad=\frac{1}{2}\left[\max _{\left\{\alpha_{0}, \alpha_{1}\right\}}[U(R+\beta S, \alpha)+U(R-\beta, \alpha)]\right] .
\end{aligned}
$$

Here the given values of the parameters will lead the individual to choose $\alpha=\alpha_{0}$ and $\beta_{I} \approx 0.350$. Thus reducing the individual's flexibility actually leads to a higher proportion of wealth invested in the risky asset.

The mathematical point made here is quite straightforward: even though the greater flexibility ensures that expression (1) will be at least as great as expression (2) for each choicc of $\beta$, it is nevertheless still possible for (1) to peak at a lower $\beta$ than does (2).

However, since the individual's insurance premia for temporal prospects are always at least as great as for identically distributed timeless

5 For these values of the parameters it follows that $\xi(c, \alpha)$ is both monotonic in $(c, \alpha)$ and concave in $c$ over the entire consumption set $R_{+}^{1} \times\left\{\alpha_{0}, \alpha_{1}\right\}$. 
prospects, this example might seem to contradict the Arrow-Pratl result that if one preference ranking over distributions exhibits higher insurance premia than another, it will also generate a lower demand for the risky asset. However, this theorem assumed that both preference rankings satisfied the expected utility axioms, and a Markowitz (1959, chs. 10, 11), Mossin (1969), and others have shown, while an expected utility maximizer's preferences over timeless prospects continue to satisfy the axioms, his or her preferences over temporal prospects in general will not. ${ }^{6}$ Yaari (1969), for example, has already provided an example where one (nonexpected utility) preference ranking assigns higher insurance premia than another, yet may generate a higher demand for risky assets. ${ }^{7}$ Viewed in this light, the contribution of the present note is to show that such pairs of rankings may be generated by looking at the same individual's preferences over timeless and temporal prospects, or equivalently, under two different degrees of flexibility. ${ }^{8}$

\section{References}

Arrow, Kenneth J., 1974, Essays in the theory of risk-bearing (North-Holland, Amsterdam). Drèze, Jacques H. and Franco Modigliani, 1972, Consumption decisions under uncertainty, Journal of Economic Theory 5, 308-355.

Eden, Benjamin, 1977, The role of insurance and gambling in allocating risk over time, Journal of Economic Theory 16, 228-246.

Epstein, Larry G., 1980, Decision making and the temporal resolution of uncertainty, International Economic Review 21, 269-283.

Epstein, Larry G. and Stuart M. Turnbull, 1980, Capital asset prices and the temporal resolution of uncertainty, Journal of Finance 35, 627-643.

${ }^{6}$ In other words, while $W(\cdot)$ is clearly a linear functional of $F(\cdot), V(\cdot)$ in general will not be. For a method of applying 'expected utility' techniques to the analysis of such general non-linear preference functionals, see Machina (1982).

7 Yaari's example [Yaari (1969, fig. 2)] occurred in his study of the property of 'declining risk aversion in wealth'. The relevant rankings are thus the individual's rankings over (timeless) gambles from two different initial wealth levels.

8 Mention should be made of two related works in the field of temporal risk. Epstein (1980) provides a general comparative statics theorem relating choice behavior to the timing of the resolution of uncertainty, and applies this result to several examples, although he does not consider the asset demand problem considered here. Epstein and Turnbull (1980) consider the effects of the timing of the resolution of uncertainty in a multiperiod capital asset pricing model. However, the particular functional form of their utility function (which has a coefficient of risk aversion which is independent of wealth or any action the individual takes) effectively rules out the possibility of the phenomenon considered here. 
Kreps, David M. and Evan L. Porteus, 1979, Temporal von Neumann-Morgenstern and induced preferences, Journal of Economic Theory 20, 81-109.

Machina, Mark J., 1982, 'Expected utility' analysis without the independence axiom, Econometrica 50, 277-323.

Markowitz, Harry M., 1959, Portfolio selection: Efficient diversification of investment (Yale University Press, New Haven, CT).

Mossin, Jan, 1969, A note on uncertainty and preferences in a temporal context, American Economic Review 59, 172-174.

Pratt, John W., 1964, Risk aversion in the small and in the large, Econometrica 32, $122-136$.

Rossman, Michael and Larry Selden, 1979, Notes on indirect cardinal utility and intertemporal choice under uncertainty, Columbia University Department of Economics discussion paper no. 48.

Spence, Michael and Richard Zeckhauser, 1972, The effect of the timing of consumption decisions and the resolution of lotteries on the choice of lotteries, Econometrica 40, 401-403.

Tisdell, Clem, 1963, Uncertainty, instability, expected profit, Econometrica 31, 243-247.

Yaari, Menahem E., 1969, Some remarks on measures of risk aversion and on their uses, Journal of Economic Theory 1, 315-329. Reprinted in Peter A. Diamond and Michael Rothschild, eds., 1978, Uncertainty in economics: Readings and exercises (Academic Press, New York). 